\title{
Uncommon manifestations of neonatal group b Streptococcus infection: case report and literature review
}

\author{
GREGOR NOSAN, DOMINIKA ZUGELJ, DARJA PARO-PANJAN \\ Department of Neonatology, Division of Pediatrics, University Medical Centre Ljubljana, Ljubljana, Slovenia
}

Corresponding author

Gregor Nosan

Department of Neonatology

Division of Pediatrics

University Medical Centre Ljubljana

Bohoriceva ulica 20, SI-1525 Ljubljana, Slovenia

Phone: +38615229274

Fax: +38615224035

E-mail: gregor.nosan@kclj.si

\section{ABSTRACT}

Streptococcus agalactiae, also called Group B Streptococcus (GBS), is a common pathogen in the neonatal period that can cause early- and late-onset infections. The most common manifestations are bacteremia without an apparent focus of infection, pneumonia and meningitis. Additionally, GBS can rarely cause early- and late-onset infections with uncommon manifestations. If they go unrecognized, they may lead to inappropriate treatment and increased neonatal morbidity and mortality. In this article, a case report of an infant with early-onset GBS bullous impetigo is presented together with a short review of other uncommon manifestations of GBS infection.

Key words: Streptococcus agalactiae, infection, newborn

\section{INTRODUCTION}

Streptococcus agalactiae, also called Group B Streptococcus (GBS), can cause earlyand late-onset infections in neonates. The most common manifestations of early-onset infections are bacteremia without any apparent focus, pneumonia and meningitis. In late-onset infections, the most typical manifestations are bacteremia without any apparent focus, pneumonia, cellulitis, osteoarthritis and meningitis. Besides these frequent and well-recognized manifestations, there are rare, atypical manifestations of early- and late-onset GBS infec- tions described in the literature. In this article, we present a case report of an infant with early-onset neonatal bullous impetigo caused by GBS and a short review of other uncommon GBS manifestations.

\section{CASE PRESENTATION}

A male infant was born at term by vaginal delivery to a healthy 36-year-old gravida after her second and uneventful pregnancy. Labor started spontaneously, the fetal membranes were artificially ruptured and the amniotic fluid was milky. The infant's Apgar scores were $7 / 7 / 9$ and his birth measures were normal. Caput succedaneum, scalp excoriations due to artificial rupture of the fetal membranes and a single large bulla, measuring $2 \mathrm{~cm}$ in diameter, filled with clear yellowish fluid, were all found parietally on the left side of the head. The physical examination was otherwise normal.

The following day, several new, similar vesicles and bullae, measuring from a few millimeters to 1 centimeter in diameter and encircled by redness of the surrounding skin, appeared near the primary lesion (figure 1).

The skin and mucous membranes were otherwise not affected. The primary bulla was punctured, and the clear yellowish content revealed the presence of multinuclear giant cells. $\mathrm{C}$ reactive protein (CRP) was slightly elevated and there was a mild pleocytosis of the cerebrospinal fluid
(CSF). Due to a strong suspicion of neonatal Herpes simplex virus (HSV) infection, parenteral therapy with acyclovir was started. However, all samples taken (vesicle fluid, blood and CSF) were negative for HSV type 1 and 2 and varicella-zoster virus (polymerase chain reaction (PCR) method). Furthermore, the mother's vaginal swab was also negative for HSV type 1 and 2 (PCR method) and her serology tests showed only the presence of specific HSV type 1 IgG antibodies.

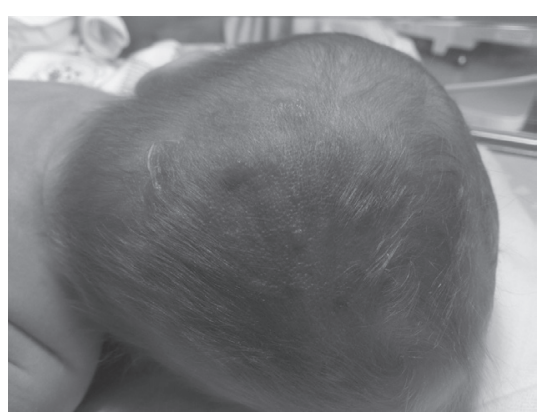

Figure 1. Vesicles and ruptured bullae parietally on the left side of the head.

The following day, as there was no clinical improvement, and as GBS was isolated from the vesicle fluid, acyclovir therapy was stopped and parenteral ampicillin therapy was instituted. Soon after that, the vesicles began to dry up, repeat CRP was negative and the skin healed without any consequences in several days. As blood and CSF cultures remained sterile, the infant received parenteral ampicillin for 7 days. No late-onset GBS infection occurred. 


\section{DISCUSSION}

This case report of neonatal bullous impetigo presents an uncommon clinical manifestation of early-onset GBS infection. As a matter of fact, the presentation was so unusual that it was mistaken for neonatal HSV infection and the appropriate treatment was consequently delayed. The distribution of vesicles on the scalp was similar to that seen in most cases of HSV infection. On the contrary, the infant was born with vesicles, which is seen in only $5 \%$ of congenital HSV infection. In most cases of congenital HSV infection, the skin and/or vesicular lesions develop over or after several days. (1) On the other hand, 60-70\% of all GBS cases occur as early-onset infection. (2)

There are only rare reports of early-onset GBS infection with skin manifestations. In 1976, Lopez et al. described a case of an infant born with skin erosions at different stages on his neck, trunk, axilla, hand and foot. GBS was isolated from the infant's skin lesions and the mother's cervix. (3) The case reported in 1993 by Kline and O’Donnell most closely resembles our patient. The authors presented a case of neonatal bullous skin lesions, caused by GBS, transmitted from the infant's mother and confirmed by skin histology. (4)

GBS can also cause other uncommon infections which are presented in table 1 and most commonly appear as late-onset infections.

Cases of uncommon soft tissue GBS infections include necrotizing fasciitis, parotitis, retropharyngeal cellulitis, prepatellar bursitis and abscess of cystic hygroma. Uncommon central nervous system manifestations of infection are cerebritis, ventriculitis, subdural empyema and oculomotor nerve paralysis, usually following GBS meningitis. Ventriculitis, however, can also be a primary infection. GBS can also cause uncommon eye infections such as neonatal ophthalmia, retrobulbar abscess and, in extremely rare cases, endophthalmitis, which is usually associated with bacteremia and meningitis. Since endophthalmitis can initially remain undiagnosed, it is very important to perform ophthalmoscopy in every infant with meningitis. Uncommon manifestation of GBS infection is also acute otitis media. This manifestation is most commonly a late-onset infection and a complication of meningitis. Heart infections such as endocarditis, especially if there is an associated congenital heart defect, can uncommonly be caused by GBS, usually following sepsis. Uncommon GBS respiratory tract infections include neonatal empyema and supraglottitis. There is a possible etiological association of earlyonset GBS sepsis with a late presentation of right-sided diaphragmatic hernia in infants, although the pathophysiological mechanism is not yet clear. Uncommon abdominal GBS infection is adrenal abscess, probably due to bacterial seeding from adrenal hemorrhage. Late-onset urinary tract infections occur occasionally, principally in infants with congenital urinary tract anomalies such as hydronephrosis and vesicoureteral reflux.

\section{CONCLUSION}

GBS is one of the most common pathogenic bacteria that cause various neonatal infections. Most clinical manifestations are well known and recognized; atypical presentations, as in the case described, are rare. However, they must be recognized to avoid misdiagnosis and inappropriate treatment.

Table 1. Uncommon clinical manifestations of Group B Streptococcal infection. (5)

\begin{tabular}{lll}
\hline Site & Early-onset infections & Late-onset infections \\
\hline Brain & Anterior fontanel herniation & Ventriculitis \\
& Cerebritis & $\begin{array}{l}\text { Anterior fontanel herniation } \\
\text { Subdural empyema } \\
\text { Oculomotor nerve paralysis }\end{array}$ \\
\hline Eye & Ophthalmia neonatorum & Endophthalmitis \\
\hline Ear & Retrobulbar abscess & \\
\hline Cardiovascular & Otitis media & Otitis media \\
\hline Respiratory tract & & Asymptomatic bacteremia \\
& & Endocarditis \\
\hline Abdomen & Pleural empyema & Supraglottitis \\
& & Diaphragmatic hernia \\
\hline Urinary tract & Adrenal abscess & Adrenal abscess \\
\hline Skin and soft tissue & Gallbladder distention & \\
& & Urinary tract infection \\
\hline & Impetigo neonatorum & Fasciitis \\
& & Breast abscess \\
& & Parotitis \\
& & Retropharyngeal cellulitis \\
& & Purpura fulminans \\
& & Bursitis \\
& & Abscess of cystic hygroma \\
\hline
\end{tabular}




\section{REFERENCES}

1. Bale JF, Miner LJ. Herpes Simplex Virus Infections of the Newborn. Curr Treat Options Neurol 2005;7:151-6.

2. Doare KL, Heath PT. An overview of global GBS epidemiology. Vaccine 2013;31:D7囚12.

3. Lopez JB, Gross P, Boggs TR. Skin lesions in association with $\beta$-hemolytic Streptococcus group B. Pediatrics 1976;58:859-61.

4. Kline A, O’Donnell E. Group B streptococcus as a cause of neonatal bullous skin lesions. Pediatr Infect Dis J 1993;12:165-6.

5. Edwards MS, Nietz V, Baker CJ. Group B Streptococcal Infections. In: Remington JS, Klein JO, Wilson CB, Nietz V, Maldonado YA, editors. Infectious diseases of the fetus and newborn infant. Philadelphia: Elsevier Saunders; 2011. p. 444-6. 